\title{
OBSERVATION OF TeV GAMMA RAYS FROM THE CYGNUS REGION WITH THE ARGO-YBJ EXPERIMENT
}

\author{
B. Bartoli ${ }^{1,2}$, P. Bernardini ${ }^{3,4}$, X. J. Bi ${ }^{5}$, C. Bleve $^{3,4}$, I. Bolognino $^{6,7}$, P. Branchini $^{8}$, A. Budano $^{8}$, \\ A. K. Calabrese Melcarne ${ }^{9}$, P. Camarri ${ }^{10,11}{ }^{2}$ Z. CaO $^{5}$, R. Cardarelli ${ }^{11}$, S. Catalanotti ${ }^{1,2}$, C. Cattaneo $^{7}$, S. Z. Chen $^{5}$, \\ T. L. Chen ${ }^{12}$, Y. Chen $^{5}$, P. Creti ${ }^{4}$, S. W. Cui ${ }^{13}$, B. Z. Dai ${ }^{14}$, G. D'Alí Staiti ${ }^{15,16}$, Danzengluobu ${ }^{12}$, M. Dattoli ${ }^{17,18,19}$, \\ I. De Mitri ${ }^{3,4}$, B. D’Ettorre Piazzoli ${ }^{1,2}$, T. Di Girolamo ${ }^{1,2}$, X. H. Ding ${ }^{12}$, G. Di Sciascio ${ }^{11}$, C. F. FenG $^{20}$, ZhaOyang FenG $^{5}$, \\ Zhenyong Feng ${ }^{21}$, F. Galeazzi ${ }^{8}$, E. Giroletti ${ }^{6,7}$, Q. B. Gou ${ }^{5}$, Y. Q. Guo ${ }^{5}$, H. H. He ${ }^{5}$, Haibing Hu ${ }^{12}$, Hongbo Hu ${ }^{5}$,

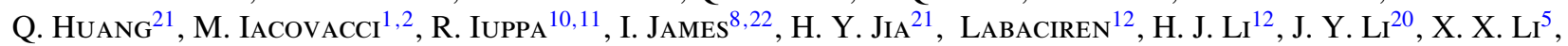

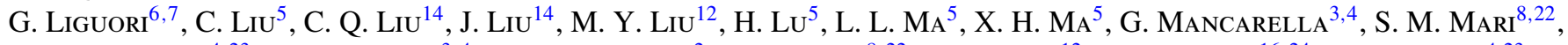 \\ G. Marsella ${ }^{4,23}$, D. Martello ${ }^{3,4}$, S. Mastroianni ${ }^{2}$, P. Montini ${ }^{8,22}$, C. C. Ning ${ }^{12}$, A. Pagliaro ${ }^{16,24}$, M. Panareo ${ }^{4,23}$, \\ B. Panico ${ }^{10,11}$, L. Perrone ${ }^{4,23}$, P. Pistilli ${ }^{8,22}$, F. Ruggieri ${ }^{8}$, P. SAlvini ${ }^{7}$, R. Santonico ${ }^{10,11}$, P. R. Shen ${ }^{5}$, X. D. Sheng ${ }^{5}$,

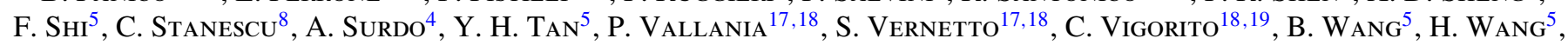

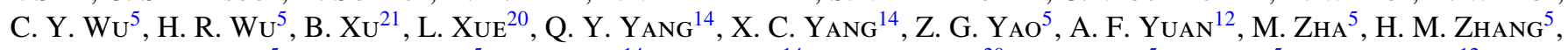

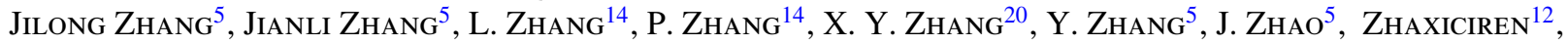 \\ ZhaXisangZhU ${ }^{12}$, X. X. ZhOU ${ }^{21}$, F. R. ZhU ${ }^{21}$, Q. Q. ZHU ${ }^{5}$, AND G. ZIZZI ${ }^{9}$ \\ (The ARGO-YBJ Collaboration) \\ ${ }^{1}$ Dipartimento di Fisica dell'Università di Napoli “Federico II," Complesso Universitario di Monte Sant'Angelo, via Cinthia, 80126 Napoli, Italy \\ ${ }^{2}$ Istituto Nazionale di Fisica Nucleare, Sezione di Napoli, Complesso Universitario di Monte Sant'Angelo, via Cinthia, 80126 Napoli, Italy \\ ${ }^{3}$ Dipartimento di Fisica dell’Università del Salento, via per Arnesano, 73100 Lecce, Italy \\ ${ }^{4}$ Istituto Nazionale di Fisica Nucleare, Sezione di Lecce, via per Arnesano, 73100 Lecce, Italy \\ ${ }^{5}$ Key Laboratory of Particle Astrophysics, Institute of High Energy Physics, Chinese Academy of Sciences, P.O. Box 918, \\ 100049 Beijing, China; chensz@ihep.ac.cn \\ ${ }^{6}$ Dipartimento di Fisica Nucleare e Teorica dell'Università di Pavia, via Bassi 6, 27100 Pavia, Italy \\ ${ }^{7}$ Istituto Nazionale di Fisica Nucleare, Sezione di Pavia, via Bassi 6, 27100 Pavia, Italy \\ ${ }^{8}$ Istituto Nazionale di Fisica Nucleare, Sezione di Roma Tre, via della Vasca Navale 84, 00146 Roma, Italy \\ ${ }^{9}$ Istituto Nazionale di Fisica Nucleare-CNAF, Viale Berti-Pichat 6/2, 40127 Bologna, Italy \\ ${ }^{10}$ Dipartimento di Fisica dell’Università di Roma “Tor Vergata,” via della Ricerca Scientifica 1, 00133 Roma, Italy \\ ${ }^{11}$ Istituto Nazionale di Fisica Nucleare, Sezione di Roma Tor Vergata, via della Ricerca Scientifica 1, 00133 Roma, Italy \\ 12 Tibet University, 850000 Lhasa, Xizang, China \\ ${ }^{13}$ Hebei Normal University, Shijiazhuang 050016, Hebei, China \\ ${ }^{14}$ Yunnan University, 2 North Cuihu Road, 650091 Kunming, Yunnan, China \\ 15 Dipartimento di Fisica e Tecnologie Relative, Università degli Studi di Palermo, Viale delle Scienze, Edificio 18, 90128 Palermo, Italy \\ ${ }^{16}$ Istituto Nazionale di Fisica Nucleare, Sezione di Catania, Viale A. Doria 6, 95125 Catania, Italy \\ ${ }^{17}$ Istituto di Fisica dello Spazio Interplanetario dell' Istituto Nazionale di Astrofisica, corso Fiume 4, 10133 Torino, Italy \\ ${ }^{18}$ Istituto Nazionale di Fisica Nucleare, Sezione di Torino, via P. Giuria 1, 10125 Torino, Italy \\ ${ }^{19}$ Dipartimento di Fisica Generale dell'Università di Torino, via P. Giuria 1, 10125 Torino, Italy \\ ${ }^{20}$ Shandong University, 250100 Jinan, Shandong, China \\ ${ }^{21}$ Southwest Jiaotong University, 610031 Chengdu, Sichuan, China \\ 22 Dipartimento di Fisica dell’Università “Roma Tre," via della Vasca Navale 84, 00146 Roma, Italy \\ ${ }^{23}$ Dipartimento di Ingegneria dell'Innovazione, Università del Salento, 73100 Lecce, Italy \\ ${ }^{24}$ Istituto di Astrofisica Spaziale e Fisica Cosmica dell'Istituto Nazionale di Astrofisica, via La Malfa 153, 90146 Palermo, Italy \\ Received 2011 October 12; accepted 2011 December 26; published 2012 January 9
}

\begin{abstract}
We report the observation of TeV $\gamma$-rays from the Cygnus region using the ARGO-YBJ data collected from 2007 November to 2011 August. Several TeV sources are located in this region including the two bright extended MGRO J2019+37 and MGRO J2031+41. According to the Milagro data set, at $20 \mathrm{TeV}$ MGRO J2019+37 is the most significant source apart from the Crab Nebula. No signal from MGRO J2019+37 is detected by the ARGO-YBJ experiment, and the derived flux upper limits at the $90 \%$ confidence level for all the events above $600 \mathrm{GeV}$ with medium energy of $3 \mathrm{TeV}$ are lower than the Milagro flux, implying that the source might be variable and hard to be identified as a pulsar wind nebula. The only statistically significant (6.4 standard deviations) $\gamma$-ray signal is found from MGRO J2031+41, with a flux consistent with the measurement by Milagro.
\end{abstract}

Key words: gamma rays: general - pulsars: individual (MGRO J2019+37, MGRO J2031+41)

Online-only material: color figures

\section{INTRODUCTION}

The Cygnus region is the brightest diffuse $\gamma$-ray emitting region in the northern sky as observed by both Fermi (Abdo et al. 2011) and EGRET (Hunter et al. 1997). Complex features have been observed in the wavelength bands of radio, infrared,
$\mathrm{X}$-rays, and $\gamma$-rays. This region is rich in potential cosmicray acceleration sites, e.g., Wolf-Rayet stars, OB associations, and supernova remnants. Recently, $24 \gamma$-ray sources, including 7 pulsars, have been detected using Fermi Large Area Telescope (LAT) two-year data within the region with $65^{\circ}<l<85^{\circ}$ and $-3^{\circ}<b<3^{\circ}$ (Abdo et al. 2011). These are considered 
candidate sources of very high energy (VHE) $\gamma$-rays. The Cygnus region is, therefore, a natural laboratory for studying the origin of cosmic rays.

Several VHE $\gamma$-ray sources have been detected within the Cygnus region in the past decade. The first was $\mathrm{TeV} \mathbf{J} 2032+4130$, discovered by the HEGRA collaboration (Aharonian et al. 2002, 2005) and confirmed by the Whipple (Konopelko et al. 2007) and MAGIC (Albert et al. 2008) experiments. Its extension is estimated to be about 0.1 . The power-law spectral index is about -2.0 and the integral flux above $1 \mathrm{TeV}$ is $3 \%-5 \%$ that of the Crab flux. MGRO J2031+41, detected by the Milagro experiment at $20 \mathrm{TeV}$ (Abdo et al. 2007a), is spatially consistent with the source TeV J2032+4130, while the measured extension is much larger with a diameter of $3.0 \pm 0.9$. This source is likely to be associated with the pulsar 2FGL J2032.2+4126, detected by Fermi (Abdo et al. 2011).

Evidence of $\mathrm{TeV}$ emission at the 4.0 standard deviation (s.d.) level after an X-ray flare from Cyg X-1 was observed by the MAGIC experiment on 2006 September 24 (Albert et al. 2006).

The source VER J2019+407 was discovered in a survey of the Cygnus region by the VERITAS experiment (Weinstein 2009). The measured extensions are $0.16 \pm 0.028$ and $0.11 \pm 0.027$ along the major and minor axes, respectively. This source is spatially coincident with the Fermi source 2FGL J2019.1+4040, which is potentially associated with a supernova remnant or a pulsar wind nebula (PWN) (Abdo et al. 2011).

During the deep VERITAS observations of the Cyg OB1 region, a point source, VER J2016+372, was discovered at the location of CTB 87 (Aliu 2011). The flux is about 1\% that of the $\mathrm{Crab}$ and the spectral index is about -2.1 from a preliminary analysis.

This region also contains the bright unidentified source MGRO J2019+37, which was detected by the Milagro experiment at $20 \mathrm{TeV}$ (Abdo et al. 2007a) and is the most significant source in the Milagro data set apart from the Crab Nebula. Its extension is $\sigma=0.32 \pm 0.12$ in a symmetric two-dimensional Gaussian (Abdo et al. 2007b), which has 68\% of the events contained in a region with an angular diameter of $1.1 \pm 0.5$ (Abdo et al. 2007a). The spectrum of this source is hard with an index of -1.83 and an exponential cutoff at $22.4 \mathrm{TeV}$ (Smith 2010). At the location of MGRO J2019+37, a 2.2 s.d. signal corresponding to $30 \%$ Crab unit was observed by the Tibet $\mathrm{AS} \gamma$ experiment (Amenomori et al. 2010). However, about 0.9 away, a possible source was detected (Amenomori et al. 2007). The MGRO J2019+37 is spatially coincident with the Fermi source 2FGL J2018.0+3626 and pulsar 2FGL J2021.0+3651 (Abdo et al. 2011). VERITAS has surveyed this region, but no emission from MGRO J2019+37 has been detected (Weinstein 2009). Recently, an in-depth observation of the Cyg OB1 region has been carried out by VERITAS, which unveiled complex TeV emission around MGRO J2019+37 (Aliu 2011).

Among the four known VHE $\gamma$-ray sources inside the Cygnus region, MGRO J2019+37 is enigmatic due to its high flux not being confirmed by other VHE $\gamma$-ray detectors. The measurement of the energy spectrum or an upper limit around several $\mathrm{TeV}$ is therefore very useful to understand the nature of the source and its emission mechanism. The ARGO-YBJ experiment is an air shower array with large field of view and can continuously monitor the northern sky. The total exposure to the Crab Nebula reaches about 1200 days and its photon flux has been detected with a statistical significance of 17 s.d. at energies around $1 \mathrm{TeV}$, which is comparable with the eight-year value of 17.2 s.d. obtained at energies around $35 \mathrm{TeV}$ by Milagro (Abdo et al. 2009). This work presents the observation results for the Cygnus region, including sources MGRO J2031+41 and MGRO J2019+37, with the ARGO-YBJ experiment.

\section{THE ARGO-YBJ EXPERIMENT}

The ARGO-YBJ experiment, located in Tibet, China, at an altitude of $4300 \mathrm{~m}$ above sea level, is the result of a collaboration among Chinese and Italian institutions and is designed for VHE $\gamma$-ray astronomy and cosmic-ray observations. The detector consists of a single layer of Resistive Plate Chambers (RPCs), which are equipped with pick-up strips $(6.75 \mathrm{~cm} \times 61.80 \mathrm{~cm}$ each). The logical OR of the signal from eight neighboring strips constitutes a logical pixel (called a "pad") for triggering and timing purposes. One hundred thirty clusters (each composed of 12 RPCs) are installed to form a carpet of about $5600 \mathrm{~m}^{2}$ with an active area of $\sim 93 \%$. This central carpet is surrounded by 23 additional clusters ("guard ring") to improve the reconstruction of the shower core location. The total area of the array is $110 \mathrm{~m} \times$ $100 \mathrm{~m}$. More details about the detector and the RPC performance can be found in Aielli et al. (2006, 2009a).

The ARGO-YBJ detector is operated by requiring the number of fired pads $\left(N_{\text {pad }}\right)$ to be at least 20 within $420 \mathrm{~ns}$ on the entire carpet detector. The high granularity of the apparatus permits a detailed space-time reconstruction of the shower profile and therefore of the incident direction of the primary particle. The arrival time of the particles is measured with a resolution of about $1.8 \mathrm{~ns}$ (Aielli et al. 2009a). In order to calibrate the 18,360 time-to-digital converter (TDC) channels, we have developed a method using cosmic ray showers (He et al. 2007). The calibration precision is $0.4 \mathrm{~ns}$ and the procedure is applied every month (Aielli et al. 2009b).

The central 130 clusters began taking data in 2006 July, and the "guard ring" was merged into the DAQ stream in 2007 November. The trigger rate is $3.5 \mathrm{kHz}$ with a dead time of $4 \%$ and the average duty cycle is higher than $86 \%$. The angular resolution, pointing accuracy, and stability of the ARGO-YBJ detector array have been thoroughly tested by measuring the shadow of the Moon in cosmic rays (Bartoli et al. 2011b). The point-spread function (PSF) is quantified using a parameter $\psi_{70}$ as the opening angle containing $71.5 \%$ of the events. For $N_{\text {pad }}>1000, \psi_{70}$ is 0.47 , while at $N_{\text {pad }} \sim 20 \psi_{70}$ becomes 2.8 (Bartoli et al. 2011a, 2011b). This measured angular resolution refers to cosmic-ray-induced air showers, and it is smaller by $30 \%-40 \%$ for $\gamma$-rays.

\section{DATA ANALYSIS}

The ARGO-YBJ data used in this analysis were collected from 2007 November to 2011 August. The total effective observation time is 1182.0 days. To achieve a good angular resolution, the event selections used in Bartoli et al. (2011a) are applied here and only events with a zenith angle less than $50^{\circ}$ are used. The total number of events after filtering used in this work is $1.97 \times 10^{11}$. The opening angle $\psi_{70}$ for events with $N_{\text {pad }}>60$ is 1.36 . In order to obtain a sky map, an area centered at the source location in celestial coordinates (right ascension and declination) is divided into a grid of $0.1 \times 0.1$ bins and filled with detected events according to their reconstructed arrival directions. To extract the excess of $\gamma$-rays from each bin, the "direct integral method" (Fleysher et al. 2004) is adopted in order to estimate the number of cosmic-ray background events in the bin. To remove the effect of cosmic-ray anisotropy on a spatial scale of $11^{\circ} \times 11^{\circ}$, a correction procedure described in Bartoli et al. (2011a) has been applied. To take into account 


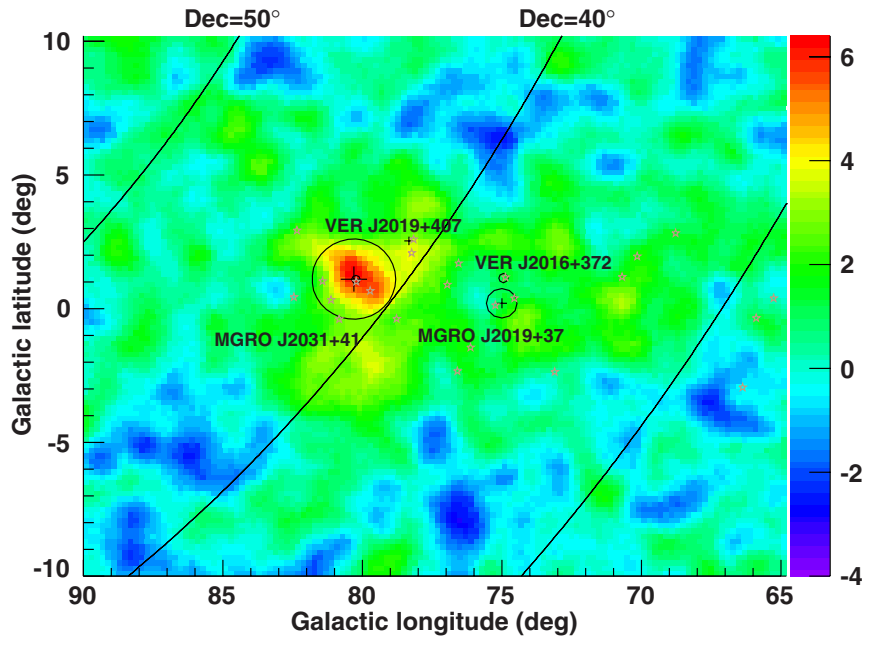

Figure 1. Significance map of the Cygnus Region as observed by the ARGOYBJ experiment. The four known VHE $\gamma$-ray source are reported. The errors on the MGRO source positions are marked with crosses, while the circles indicate their intrinsic sizes (Abdo et al. 2007a, 2007b). The cross for VER J2019+407 indicates its extension (Weinstein 2009). The source VER J2016+372 is marked with small circles without position errors. The small circle within the errors of MGROJ2031+41 indicates position and extension of the source TeV J2032+4130 as estimated by the MAGIC collaboration (Albert et al. 2008). The open stars mark the location of the $24 \mathrm{GeV}$ sources in the second Fermi-LAT catalog.

(A color version of this figure is available in the online journal.)

the PSF of the ARGO-YBJ detector, the events in a circular area centered on the bin with an angular radius of $1.3 \psi_{70}$ are summed together with a weight of the Gaussian-shaped PSF. Equation (17) in $\mathrm{Li} \& \mathrm{Ma}$ (1983) is used to estimate the significance of the excess in each bin.

With this data analysis, the significance of the excess observed from the direction of the Crab Nebula is 17 s.d., which indicates that the cumulative 5 s.d. sensitivity of ARGO-YBJ has reached 0.3 Crab unit for point sources (Cao \& Chen 2011). For an extended source with a symmetric two-dimensional Gaussian shape with $\sigma=0.32$, the sensitivity is degraded by a factor of $11 \%$.

\section{RESULTS AND DISCUSSION}

The significance map of the Cygnus region as observed by ARGO-YBJ using events with $N_{\text {pad }}>20$ is shown in Figure 1. For comparison, the 4 known $\mathrm{TeV}$ sources and $24 \mathrm{GeV}$ sources in the second Fermi-LAT catalog are marked in the figure. An excess is observed over a large part of the Cygnus region, which indicates a possible diffuse $\gamma$-ray emission. An analysis of the diffuse $\gamma$-ray emission using ARGO-YBJ data can be found in Ma (2011). The highest significance value is 6.4 s.d. at (307.85, 41.75 ), consistent with the position of VHE sources MGRO $\mathrm{J} 2031+41$ and $\mathrm{TeV} \mathbf{J} 2032+4130$. No evidence of an emission above 3 s.d. is found at the location of MGRO J2019+37.

\section{1. $M G R O J 2031+41$}

The intrinsic extension of MGRO J2031+41 is determined by fitting the distribution of $\theta^{2}$ for the events exceeding the background as shown in Figure 2, where $\theta$ is the angular distance of each event to the position of $\mathrm{TeV} \mathrm{J} 2032+4130$. Only events with $N_{\text {pad }}>60$ are used in this fit, where $N_{\text {pad }}$ is the number of fired pads. To fit the data, a set of $\gamma$-rays is generated taking into account the spectral energy distribution

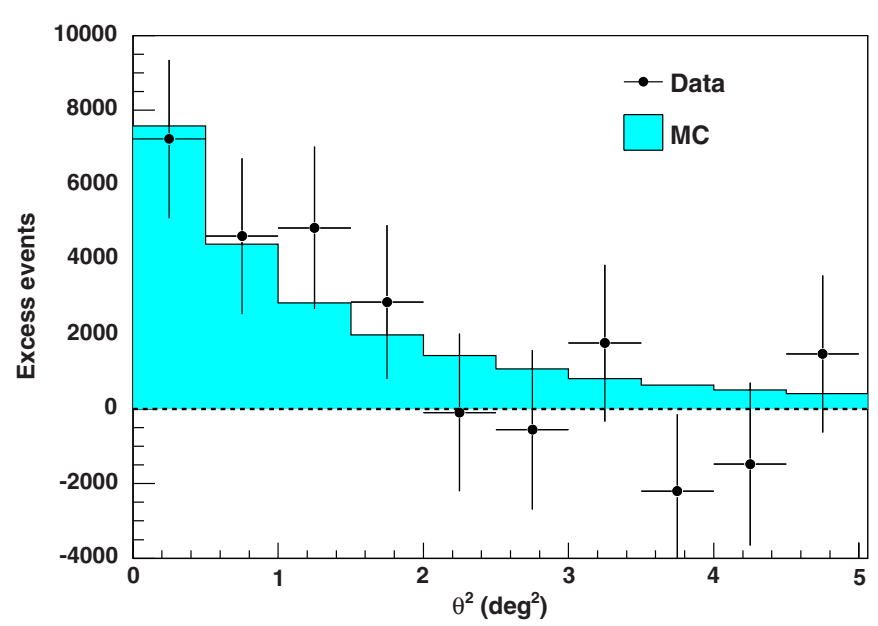

Figure 2. Distribution of $\theta^{2}$ for the number of excess events around $\mathrm{TeV}$ $\mathbf{J} 2032+4130$. The filled region is the best fit to simulated data.

(A color version of this figure is available in the online journal.)

(SED), the intrinsic source extension, and the detector PSF. The extension is estimated, by minimizing the $\chi^{2}$ between the data and the generated events, between $0^{\circ}$ and $1^{\circ}$ with steps of 0 . 1 . Assuming the background spectral index -2.8 , the intrinsic extension is determined to be $\sigma_{\text {ext }}=\left(0.2_{-0.2}^{+0.4}\right)^{\circ}$. It is found that the dependence on the SED is negligible within the uncertainties. This result is consistent with the estimation by the MAGIC and HEGRA experiments, i.e., $0.083 \pm 0.030$ and $0.103 \pm 0.025$, respectively.

Assuming an intrinsic extension $\sigma_{\text {ext }}=0.1$, we estimate the spectrum of MGRO J2031+41 using the ARGO-YBJ data by a conventional fitting method described in Bartoli et al. (2011a). In this procedure, the expectation function is generated by sampling events in the energy range from $10 \mathrm{GeV}$ to $100 \mathrm{TeV}$ and taking into account the detailed ARGO-YBJ detector response to the events assuming a power law with its spectral index as a parameter. We define four intervals with $N_{\text {pad }}$ of 60-99, 100-199, 200-499, and $\geqslant 500$. The best fit to the SED is shown in Figure 3. The differential flux $\left(\mathrm{TeV}^{-1} \mathrm{~cm}^{-2} \mathrm{~s}^{-1}\right)$ in the energy range from $0.6 \mathrm{TeV}$ to $7 \mathrm{TeV}$ is

$$
\frac{d N}{d E d A d t}=(1.40 \pm 0.34) \times 10^{-11}\left(\frac{E}{1 \mathrm{TeV}}\right)^{-2.83 \pm 0.37}
$$

The integral flux is $31 \%$ that of the Crab at energies above $1 \mathrm{TeV}$, which is higher than the flux of TeV J2032+4130 as determined by the HEGRA and MAGIC experiments, i.e., $5 \%$ and $3 \% \mathrm{Crab}$ units, respectively. However, this measurement is in agreement with the Milagro new result (Bonamente et al. 2011), also shown in Figure 3.

The reason for the discrepancy between the fluxes measured by Cherenkov telescopes and extensive air shower arrays is still unclear. A contribution is expected from the diffuse $\gamma$-ray flux produced by cosmic rays interacting with matter in the Galaxy plane. According to the measurement of diffuse $\gamma$-ray flux from the Cygnus region using ARGO-YBJ data (Ma 2011), this contribution to the measured flux from MGRO J2031+41 at energy above $1 \mathrm{TeV}$ is about $10 \%$. Moreover, an estimate of the systematic error is described in Aielli et al. (2010). With an incomplete list of the sources of systematics, such as the time resolution variation, event rate variation with environment parameters, and pointing error, the error is found to be less than $30 \%$. Due to the limited angular resolution of the ARGO-YBJ 


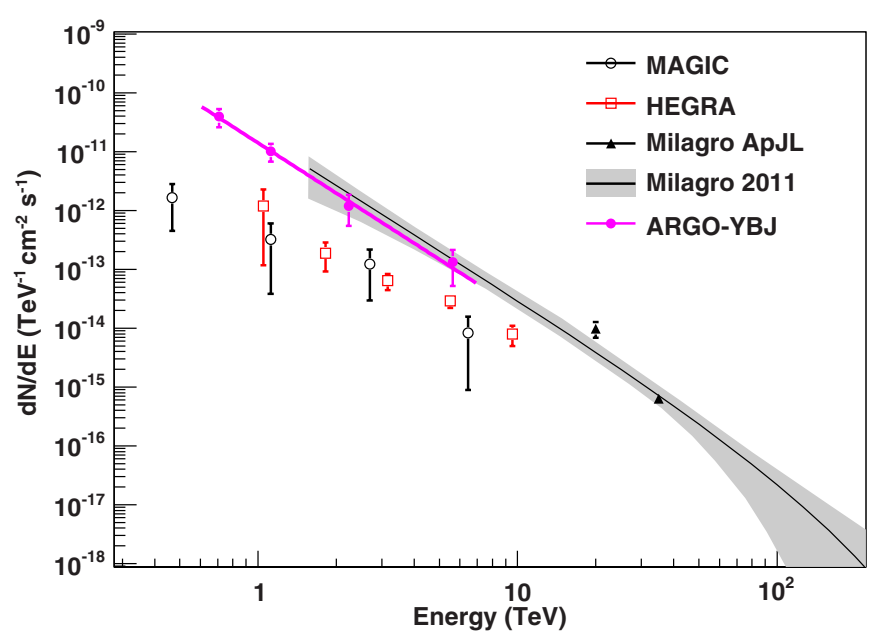

Figure 3. Energy density spectrum from TeV J2032+4130/MGRO J2031+41 as measured by the ARGO-YBJ experiment (solid magenta line). The spectral measurements of HEGRA (Aharonian et al. 2005) and MAGIC (Albert et al. 2008) are also reported for comparison. The solid line and shaded area indicate the differential energy spectrum and the $1 \mathrm{s.d}$. error region as recently determined by the Milagro experiment (Bonamente et al. 2011). The two triangles give the previous flux measurements by Milagro at $20 \mathrm{TeV}$ (Abdo et al. 2007a) and $35 \mathrm{TeV}$ (Abdo et al. 2009).

(A color version of this figure is available in the online journal.)

detector, nearby sources could contribute to the flux. For TeV $\mathrm{J} 2032+4130$, this contribution must be very small, because there is no source in the $3^{\circ} \times 3^{\circ}$ field of view as seen by HEGRA and MAGIC except TeV J2032+4130 itself (Aharonian et al. 2005; Albert et al. 2008). Thus, the contribution from diffuse $\gamma$-ray emission, nearby sources, and systematic uncertainty are not enough to explain the discrepancy.

\subsection{MGRO J2019+37}

No excess above 3 s.d. is detected inside the Cyg OB1 region, even considering extended sources. Taking into account the position uncertainty reported by Milagro, the bin with the maximum significance within 0.3 from MGRO J2019+37 is used to estimate the upper limits. The flux upper limits at the 90\% confidence level (c.1.) are shown in Figure 4 assuming the SED reported in Smith (2010) and the extension $\sigma=0.32$ given in Abdo et al. (2007b), respectively. At energies above $5 \mathrm{TeV}$, the ARGO-YBJ exposure is still insufficient to reach a firm conclusion. Regarding the emission at lower energies, the ARGO-YBJ observation does not confirm the spectrum determined by Milagro (Smith 2010). Taking into account that the two observations differ in time by several years, this discrepancy might indicate a variation in the $\gamma$-ray flux of the source.

The VERITAS experiment carried out a fine scanning of the Cygnus region. With a sensitivity of about $10 \%$ Crab unit, there was no significant signal found in the direction of MGRO J2019+37 (Weinstein 2009). With a deeper survey corresponding to a sensitivity of about $1 \%$ Crab unit, some faint sources were found in this region (Aliu 2011). The estimated flux is much weaker than that determined by the Milagro experiment.

Considering the source extension $\sigma=0.32 \pm 0.12$ and the distance of the Cygnus region $1-2 \mathrm{kpc}$, the source radius is estimated to be $4-15 \mathrm{pc}$, implying that the variation timescale should be longer than 13-49 years. The observation by the ARGO-YBJ experiment is about five years later than that by

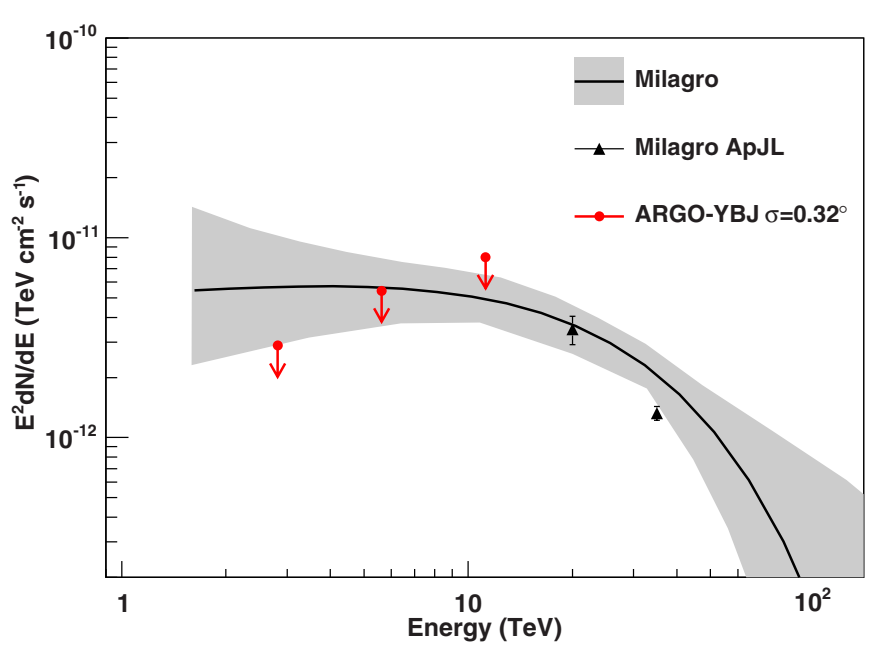

Figure 4. Upper limits to the flux from MGRO J2019+37 derived by the ARGOYBJ experiment adopting the spectrum given in Smith (2010). The extension is assumed to be $\sigma=0.32$ as given in Abdo et al. (2007b). The solid line and shaded area indicate the differential energy spectrum and the 1 s.d. error region as determined by the Milagro experiment (Smith 2010). The two triangles give the previous flux measurements by Milagro at $20 \mathrm{TeV}$ (Abdo et al. 2007a) and $35 \mathrm{TeV}$ (Abdo et al. 2009).

(A color version of this figure is available in the online journal.)

Milagro. A flux variation over the whole extended region cannot be completely excluded. If the flux variation were dominated by a smaller region in the source area, the picture could be more reasonable. In such a scenario, however, identifying MGRO J2019+37 as a PWN could be a dilemma because it otherwise should have a steady flux.

\section{CONCLUSIONS}

Since 2007 November the ARGO-YBJ experiment has been monitoring with high duty cycle the northern sky at $\mathrm{TeV}$ photon energies. Using data up to 2011 August, we have observed the Cygnus region, inside which two bright VHE extended $\gamma$-ray sources have been detected by the Milagro experiment. An excess with statistical significance of 6.4 s.d. is detected from the direction of MGRO J2031+41, consistent with the Milagro observation, but with a flux higher than that measured by HEGRA and MAGIC. The source location and extension are, however, consistent with those of $\mathrm{TeV} \mathrm{J} 2032+4130$. It is not easy to assess the origin of this discrepancy. No signal from MGRO J2019+37 is detected, and the derived upper limits at the $90 \%$ c.l. are lower than the Milagro flux at energies below $5 \mathrm{TeV}$. This result could be explained by invoking a source variability, making it difficult in that case to identify the source as a PWN. In conclusion, further observations and attention to the Cygnus region are needed since it is found to be complex in the VHE domain.

This work is supported in China by NSFC (No. 10120130794), the Chinese Ministry of Science and Technology, the Chinese Academy of Sciences, the Key Laboratory of Particle Astrophysics, CAS, and in Italy by the Istituto Nazionale di Fisica Nucleare (INFN).

We also acknowledge the essential support of W. Y. Chen, G. Yang, X. F. Yuan, C. Y. Zhao, R. Assiro, B. Biondo, S. Bricola, F. Budano, A. Corvaglia, B. D’Aquino, R. Esposito, A. Innocente, A. Mangano, E. Pastori, C. Pinto, E. Reali, F. Taurino, and 
A. Zerbini, in the installation, debugging, and maintenance of the detector.

\section{REFERENCES}

Abdo, A. A., Allen, B., Berley, D., et al. 2007a, ApJ, 664, L91

Abdo, A. A., Allen, B., Berley, D., et al. 2007b, ApJ, 658, L33

Abdo, A. A., Allen, B. T., Aune, T., et al. 2009, ApJ, 700, L127

Abdo, A. A., et al. 2011, arXiv:1108.1435v1

Aharonian, F., Akhperjanian, A., Beilicke, M., et al. 2002, A\&A, 393, L37

Aharonian, F., Akhperjanian, A., Beilicke, M., et al. 2005, A\&A, 431, 197

Aielli, G., Bacci, C., Bartoli, B., et al. 2009a, Nucl. Instrum. Methods Phys. Res. A, 608, 246

Aielli, G., Assiro, R., Bacci, C., et al. 2006, Nucl. Instrum. Methods Phys. Res. A, 562, 92

Aielli, G., Bacci, C., Bartoli, B., et al. 2009b, Astropart. Phys., 30, 287

Aielli, G., Bacci, C., Bartoli, B., et al. 2010, ApJ, 714, L208

Albert, J., Aliu, E., Anderhub, H., et al. 2006, ApJ, 665, L51

Albert, J., Aliu, E., Anderhub, H., et al. 2008, ApJ, 675, L25

Aliu, E. 2011, in Proc. 32nd ICRC, in press (available at http://icrc2011.ihep. ac.cn)
Amenomori, M., Bi, X. J., Chen, D., et al. 2007, in Proc. 30th ICRC, ed. R. Caballero et al. (Mexico City: Univ. Nacional Autónoma de México), 695 (available at http://www.icrc2007.unam.mx/proceedings)

Amenomori, M., Bi, X. J., Chen, D., et al. 2010, ApJ, 709, L6

Bartoli, B., Bernardini, P., Bi, X. J., et al. 2011a, ApJ, 734, 110

Bartoli, B., Bernardini, P., Bi, X. J., et al. 2011b, Phys. Rev. D, 84, 022003

Bonamente, E., Galbraith-frew, J., \& Hüntemeye, P. 2011, in Proc. 32nd ICRC, in press (available at http://icrc2011.ihep.ac.cn)

Cao, Z., \& Chen, S. Z. 2011, in Proc. 32nd ICRC, in press (arXiv:1110.1809v1)

Fleysher, R., Fleysher, L., Nemethy, P., et al. 2004, ApJ, 603, 355

He, H. H., Bernardini, P., Calabrese Melcarne, A. K., \& Chen, S. Z. 2007, Astropart. Phys., 27, 528

Hunter, S. D., Bertsch, D. L., Catelli, J. R., et al. 1997, ApJ, 481, 205

Konopelko, A., Atkins, R. W., Blaylock, G., et al. 2007, ApJ, 658, 1062

Li, T. P., \& Ma, Y. Q. 1983, ApJ, 272, 317

Ma, L. L. 2011, in Proc. 32nd ICRC, in press (available at http://icrc2011. ihep.ac.cn)

Smith, A. J. 2010, in Proc. Fermi Symposium (available at http://www.slac. stanford.edu/econf/C0911022)

Weinstein, A. 2009, in Proc. Fermi Symposium (available at http://www.slac. stanford.edu/econf/C0911022) 